\title{
Natural convection heat transfer in V-trough solar concentrators
}

\author{
T.N. Anderson ${ }^{1 *}$ \\ ${ }^{1}$ School of Engineering, Auckland University of Technology, Auckland 1142, New Zealand
}

\begin{abstract}
The use of V-trough concentrators offers a simple approach to increasing the radiation incident on a solar receiver; this is particularly useful for increasing the temperature in thermal systems. However in order to accurately predict the performance of thermal systems under concentrated radiation, there is a need to understand the heat loss from them. This study experimentally shows that, for a laterally and longitudinally inclined enclosed V-trough concentrator, the natural convection heat loss can be predicted using an equation of the form $N u=a R a^{\mathrm{b}}$ for Rayleigh numbers between $2 \times 10^{3}$ and $1 \times 10^{8}$. It is suggested that the relationship is a good representation of heat transfer in enclosed V-trough concentrators under most practical orientations, and thus lends itself for implementation into design models for V-trough concentrators.
\end{abstract}

Keywords: heat transfer, solar concentrator, V-trough

\section{Introduction}

The use of flat reflective elements to increase the radiation incident on a solar absorber has a long history. In one of the pioneering studies Tabor (1966) demonstrated a variable geometry "V-trough" collector which was found to offer the ability of achieving higher temperatures than fixed flat plate collectors.

Bannerot and Howell (1977 and 1979) furthered the understanding of V-trough style collectors by the development of a series of design nomographs that could be used in the determination of the performance of such collectors. They suggested that such collectors could offer an effective approach to reducing the cost of solar absorbers, particularly in locations with a high fraction of direct radiation. In a parallel study Mannan and Bannerot (1978) extended the idea of a single facet reflector V-trough to a two-facet design, which they found offered a compromise between a V-trough and a compound parabolic concentrator (CPC). In subsequent studies Puri (1981) and Sorour et al (1991) also showed that the performance of V-trough receivers was dependent

\footnotetext{
* Corresponding Author: Tel.: +64 99219999 x8075

E-mail address: timothy.anderson@aut.ac.nz
} 
not only on the concentration ratio and acceptance angles but also on the length of the trough. More recently Fraindenraich (1998) and Martin and Ruiz (2008) explored the optical properties of V-trough collectors, thus building on the foundations set by Bannerot and Howell.

Despite something of a hiatus during the late 1980's and 1990's, the use of V-trough collectors has started to receive renewed interest in light of increasing demand for energy. Recently Chong et al (2011) and Kostic and Pavlovic (2012) explored the use of V-trough collectors for water heating. Similarly, Riffat and Mayere (2013) suggested that V-troughs offered a promising source of heat for solar desalination applications while Wu et al (2012) utilised a V-trough shaped enclosure in conjunction with a Fresnel lens photovoltaic concentrator.

Though all the preceding studies mentioned have explored the potential for increasing the radiation on the absorber surface, there are significantly fewer studies that have examined the heat balance of the absorber, in generalizable terms, and of the heat loss in a V-trough enclosure due to natural convection in particular. Iyican et al (1980 and 1981) conducted one of the most detailed studies of V-trough style enclosure. In their work they characterised the heat loss of a V-trough enclosure inclined laterally to the horizontal for Rayleigh numbers in the range $2 \mathrm{x}$ $10^{3}-5 \times 10^{7}$ using a series of correlations of the form $\mathrm{Nu}=\mathrm{a} \mathrm{Ra}{ }^{\mathrm{b}}$. They suggested that this approach was more suitable than attempting to utilise existing correlations for parallel plates that had been developed at the time. Furthermore, in their later study, they noted that the heat transfer appeared to be an independent of the inclination angle of their enclosure.

In a later study Meyer et al (1982) also explored the convective losses in laterally inclined Vtrough enclosures suggesting that the heat loss was similar to that of flat plate collectors. Furthermore they suggested that concentration ratio could be used as an indicator of heat transfer in such collectors. Ronnelid and Karlsson (1996) also examined heat loss in laterally inclined V-trough enclosures, and though examining the issue of natural convection, they suggested addressing the materials used in these collectors to reduce radiation losses.

It is interesting to note that all these studies concentrate on laterally inclined V-trough enclosures. However in two recent studies on natural convection in CPC collector cavities, Singh and Eames (2011 and 2012) found that inclination to the horizontal in the longitudinal direction also had a marked influence on the heat loss. As such, there is a need to determine if this applies to V-trough enclosures and to develop a single generalizable relationship to describe 
the heat loss due to natural convection in such enclosures when inclined laterally and longitudinally.

\section{Experimental Method}

For this study, a V-trough style enclosure was fabricated to allow measurements of the natural convection heat transfer coefficients to be conducted while laterally or longitudinally inclined. In their preceding study, Iyican et al (1980 and 1981) used a relatively small enclosure (each Vtrough having an absorber $25.4 \mathrm{~mm} \times 610 \mathrm{~mm}$ and a height of $77 \mathrm{~mm}$ ) and varied the air pressure to modify the density and hence Rayleigh number in their study. In this study the enclosure was much larger (an absorber 200mm x 1200mm and a cavity height of $285 \mathrm{~mm}$ ) and the Rayleigh number was varied by increasing the temperature gradient. While doing this, the enclosure inclination was varied between 0 and $90^{\circ}$ laterally and 0 and $60^{\circ}$ longitudinally in $30^{\circ}$ increments (Figure 1).

A temperature gradient was generated in the enclosure by a foil resistance heater (375W nominal) adhesively bonded to a polished $2 \mathrm{~mm}$ thick aluminium plate. This heater formed what would be the heated absorber surface at the base of the V-trough enclosure. The use of aluminium plate allows the temperature profile of the heated surface to be relatively uniform across its area, this is achieved because aluminium has a high thermal conductivity and minimal resistance to heat transfer through it. The mean temperature of the heater was determined from a series of six copper-constantan (T-type) thermocouples ( $\pm 0.3 \mathrm{~K})$ placed uniformly over its surface area. Subsequently the heater was insulated with approximately $50 \mathrm{~mm}$ of mineral wool fibre insulation (R2.2) and backed by a sheet of 20mm plywood.

The angled sides of the V-trough were also made from polished aluminium sheet to minimise radiant heat exchange between the heater and them. These sides were also insulated with approximately $100 \mathrm{~mm}$ of the mineral wool fibre insulation held in place with $20 \mathrm{~mm}$ plywood. The enclosure ends were fabricated from a single layer of $20 \mathrm{~mm}$ plywood.

Additionally, the top cover was also constructed from aluminium plate to ensure that there would be minimal thermal resistance across this surface. This top cover aluminium plate was cooled by a fan providing a free stream velocity of approximately $4 \mathrm{~m} / \mathrm{s}$. This step was done to ensure that the majority of the heat transfer away from the enclosure occurred via this surface. Three thermocouples were attached to the cover and an additional thermocouple was used to measure the ambient temperature. Finally, the thermal breaks between the surfaces of the 
enclosure were sealed with high temperature duct tape to ensure that no air leakage could occur, thus leading to parasitic heat losses.

In varying the Rayleigh number in the enclosure, the temperature difference across the enclosure was controlled using a variable power transformer (Variac) connected to the resistance heating element. The power being drawn by the heater was measured using a single phase Powertech power meter accurate to $\pm 2 \%$. To determine the temperature gradient in the enclosure (between the heater and top cover) the thermocouples were connected to two Picolog TC-08 eight channel thermocouple data acquisition system and recorded by a computer via the USB interface. The configuration of the experimental measurement system and instrumentation is illustrated schematically in Figure 2.

Finally, to accurately determine the heat transfer coefficient within the enclosure it was necessary to allow the system to reach steady state conditions. To do this, the heater power was set and the ambient, cover and heater temperatures were monitored. When the variation of the temperature difference between the heater and the cover was not more than $0.6 \mathrm{~K}$ over a 30 minute period, it was assumed that the system had reached a steady state. Subsequently, the readings taken during this period were used to determine the natural convection heat transfer coefficient.

\section{Analysis}

The natural convection heat transfer coefficient for the heated surface $\left(h_{c}\right)$ in the enclosure can be determined from Newton's law of cooling as shown in Equation 1.

$$
h_{c}=\frac{Q_{\text {convection }}}{A_{p}\left(T_{p}-T_{c}\right)}
$$

Where $Q_{\text {convection }}$ is the rate heat is transferred from the heated to the cooled wall by convection based on the temperature difference between the average heater temperature $\left(T_{p}\right)$ and the cover temperature $\left(T_{c}\right)$ and for a heater area $\left(A_{p}\right)$.

Now if one was to consider that all sides of the enclosure were extremely well insulated, except the cooled cover, determination of the natural convection heat transfer coefficient would be a relatively straight-forward process. Under such conditions, at steady state, the natural 
convection heat transfer coefficient would be equal to the overall heat loss coefficient $(U)$ as shown in Equation 2, where $Q_{e}$ is the electrical power input.

$$
U=\frac{Q_{e}}{A_{p}\left(T_{p}-T_{c}\right)}
$$

However, because all the sides other than the cooled top cover were not adiabatic, a full energy balance is needed to identify the heat that is being transferred by convection. For the V-trough enclosure used in the experiment, the energy balance is given by Equation 3:

$$
Q_{e}-Q_{\text {end,cond }}-Q_{\text {back,cond }}-Q_{\text {side,cond }}-Q_{\text {rad }}=Q_{\text {convection }}
$$

where $Q_{\text {end,cond }}$ is the rate of conductive heat transfer though the ends of the enclosure at a bulk enclosure temperature $\left(T_{e}\right), Q_{b a c k, c o n d}$ is the rate of conductive heat transfer from the heaters rear surface, $Q_{\text {side,cond }}$ is the rate of conductive heat transfer through the inclined sides at a bulk enclosure temperature, and $Q_{r a d}$ is the rate of radiation heat transfer.

The remaining term, $Q_{\text {convection }}$, is the rate heat is transferred from the heated to the cooled wall by convection as described in Equation 1. If one were to consider a resistance network analysis of the system, it would be seen that the thermal resistance associated with this term is the sum of the natural convection resistance and the conduction resistance through the aluminium cover wall. However, because the thermal resistance of the aluminium wall is small relative to that of the natural convection, its influence on the calculation of the heat transfer coefficient can be neglected.

The determination of the conductive heat losses from the end, side and back walls (i.e. $Q_{\text {end,cond, }}$ $\left.Q_{\text {back,cond }}, Q_{\text {side,cond }}\right)$ is a simple application of Fourier's law over the area of the wall of interest considering both the thermal conductivity of the wall and its thickness. The rate of radiation heat transfer in the enclosure, however, is somewhat more complicated. For the case of the Vtrough enclosure, heat transfer by radiation between the heater and top plate can be considered to be negligible given that they were made from polished aluminium plate with a very low emissivity $\left(\varepsilon_{p} \approx 0.06\right.$ ). Also the inclined sides of the $\mathrm{V}$-trough also had low emissivity and because of the high level of insulation, were essentially adiabatic, and in this regard could be discounted from any radiation calculations. Finally the view factor from the heated surface to the end walls and the area of the walls of the cavity were relatively small, thus reducing the potential for large amounts of radiation heat transfer (Siegel and Howell, 2002). 
To confirm that the influence of radiation would be small in light of the view factors and small area, it was assumed that the enclosure could be treated as a simple two-surface enclosure where the radiation heat transfer could be determined using Equation 4. The first surface being the heater with an area $\left(A_{p}\right)$ and is made of aluminium with emissivity $\left(\varepsilon_{p}\right)$. The second surface considered is wood with an emissivity $\left(\varepsilon_{w}\right)$, an area equal to the sum of the areas of the ends of the enclosure $\left(A_{w}\right)$ and a view factor $(F)$ between the two surfaces equal to unity.

$$
Q_{\text {rad }}=\frac{\sigma\left(T_{p}^{4}-T_{e}^{4}\right)}{\frac{1-\varepsilon_{p}}{A_{p} \varepsilon_{p}}+\frac{1-\varepsilon_{w}}{A_{w} \varepsilon_{w}}+\frac{1}{A_{p} F}}
$$

Based on this conservative treatment of the rate of radiation heat transfer $\left(Q_{r a d}\right)$ it was found that radiation heat transfer accounted for less than $5 \%$ of the heat being transferred between the cavity elements. On this basis it was assumed that in reality it would be essentially negligible. Therefore, based on the energy balance, it was possible to determine the heat transfer by convection between the heated and cooled surfaces as described in Equation 1

\section{Results}

To determine magnitude of the natural convection heat transfer coefficients in the V-trough, the heater power was varied such that the mean heater temperatures were between $45^{\circ} \mathrm{C}$ and $110^{\circ} \mathrm{C}$ under steady state conditions for each lateral and longitudinal inclination angle. For this study the range of plate temperatures equated to Rayleigh Numbers in the range of $4 \times 10^{7}$ to $1 \times 10^{8}$, where the characteristic length was taken to be the vertical height of the enclosure, and the physical properties were taken at the mean enclosure temperature. In Figure 3 it can be seen that there exists a relationship between the Nusselt number and Rayleigh number, for all test conditions, that can be represented in the general form $N u=a a^{b}$.

Now, in a previous study Iyican et al (1981) proposed the use of Eqn.5 for determining heat loss in laterally inclined trapezoidal enclosures with Rayleigh numbers between $2 \times 10^{3}$ and $5 \times 10^{7}$. Therefore, it was decided to see if this correlation was also valid for Rayleigh number of up to $1 \times 10^{8}$ and also for longitudinally inclined enclosures.

$$
N u=0.13 R a^{0.345}
$$


In Figure 4 the Nusselt number from the experiment is compared with that calculated using Iyican et al (1981) relationship. It can be seen that although Eqn. 5 was originally developed for laterally inclined enclosures, it is actually able to provide a reasonable prediction of the heat transfer at both increased Rayleigh numbers and in longitudinally inclined enclosures (Pearson's correlation coefficient $r=0.7$ ). This suggests that the correlation is well suited for use over a much wider range of conditions than which it was initially developed for and, more importantly, is a suitable generalised representation of heat transfer in enclosed V-trough concentrators under many practical orientations.

\section{Conclusion}

V-trough concentrators offer a simple approach to increasing the radiation incident on a solar receiver; this is particularly useful for increasing the temperature in thermal systems. In order to accurately predict the performance of thermal systems under concentrated radiation, there is a need to understand the heat loss from them.

This study has shown that for an enclosed V-trough concentrator, the natural convection heat loss could be predicted by extending the range of applicability of Iyican et al (1981) relationship for Rayleigh numbers to between $2 \times 10^{3}$ and $1 \times 10^{8}$. Furthermore it was shown that the correlation could be applied to both laterally and longitudinally inclined enclosures. This suggests that the relationship is a good representation of heat transfer and, given its simplicity, lends itself well for implementation into design models of V-trough concentrators.

\section{References}

Bannerot, R.B., Howell, J.R., 1977, The effect of non-direct insolation on the radiative performance of trapezoidal grooves used as solar energy collectors, Solar Energy, 19 (5), 549553

Bannerot, R.B., Howell, J.R., 1979, Predicted daily and yearly average radiative performance of optimal trapezoidal groove solar energy collectors, Solar Energy, 22 (3), 229-234

Bejan, A., 2004, Convection heat transfer, Wiley, New York

Chong, K.K., Chay, K.G., Chin K.H., 2012, Study of a solar water heater using stationary Vtrough collector, Renewable Energy, 39 (1), 207-215

Fraidenraich, N, 1998, Design procedure of V-trough cavities for photovoltaic systems, Progress in Photovoltaics: Research and Applications, 6 (1), 43-54

Iyican, L., Witte, L.C., Bayazitoglu, Y., 1980, An experimental study of natural convection in trapezoidal enclosures, ASME Journal of Heat Transfer, 102, 648-653 
Iyican, L., Witte, L.C., Bayazitoglu, Y., 1981, Natural convection and sidewall losses in trapezoidal groove collectors, ASME Journal of Heat Transfer, 103, 167-172

Kostić, L.T., Pavlović, Z.T., 2012, Optimal position of flat plate reflectors of solar thermal collector, Energy and Buildings, 45, 161-168

Mannan, K.D., Bannerot, R.B., 1978, Optimal geometries for one- and two-faced symmetric side-wall booster mirrors, Solar Energy, 21 (5), 385-391

Martín, N., Ruiz, J. M., 2008, Optical performance analysis of V-trough PV concentrators, Progress in Photovoltaics: Research and Applications, 16, 339-348

Meyer, B.A., Mitchell, J.W., El-Wakil, M.M., 1982, Convective heat transfer in vee-trough linear concentrators, Solar Energy, 28 (1), 33-40

Puri, V.M., 1981, The effect of length on absorption for a trapezoidal groove collector, Solar Energy, 27 (6), 463-467

Riffat, S., Mayere, A., 2013, Performance evaluation of v-trough solar concentrator for water desalination applications, Applied Thermal Engineering, 50 (1), 234-244

Rönnelid, M., Karlsson, B., 1996, Experimental investigation of heat losses from lowconcentrating non-imaging concentrators, Solar Energy, 57 (2), 93-109

Siegel, J., Howell, J., 2002, Thermal radiation heat transfer, Taylor and Francis, New York Singh, H., Eames, P.C., 2011, A review of natural convective heat transfer correlations in rectangular cross-section cavities and their potential applications to compound parabolic concentrating (CPC) solar collector cavities, Applied Thermal Engineering, 31 (14-15), 21862196

Singh, H., Eames, P.C., 2012, Correlations for natural convective heat exchange in CPC solar collector cavities determined from experimental measurements, Solar Energy, 86 (9), 24432457

Sorour, M.M., Abdou, A.A.M., Mahmoud, R.A., 1991, Design of a stationary finite length Vtrough solar collector, International Journal of Solar Energy, 10, 63-81

Tabor, H., 1966, Mirror boosters for solar collectors, Solar Energy, 10 (3), 111-118

Wu, Y., Eames, P., Mallick, T., Sabry, M., 2012, Experimental characterisation of a Fresnel lens photovoltaic concentrating system, Solar Energy, 86 (1), 430-440 
Fig. 1: Laterally $(\theta)$ and longitudinally $(\phi)$ inclined trapezoidal enclosures

Fig. 2: Schematic representation of experimental apparatus

Fig. 3: Nusselt number v Rayleigh number

Fig. 4: Relationship between Nusselt number from Iyican et al (1981) correlation versus measured Nusselt number 

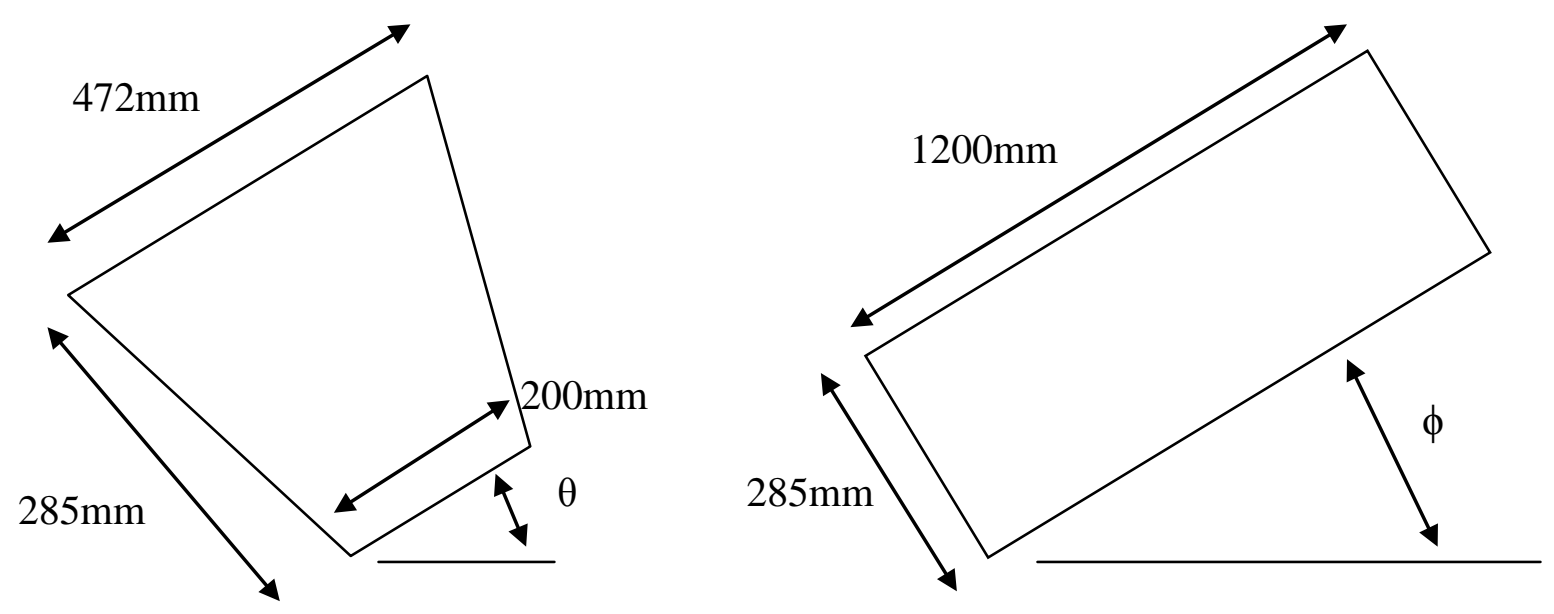


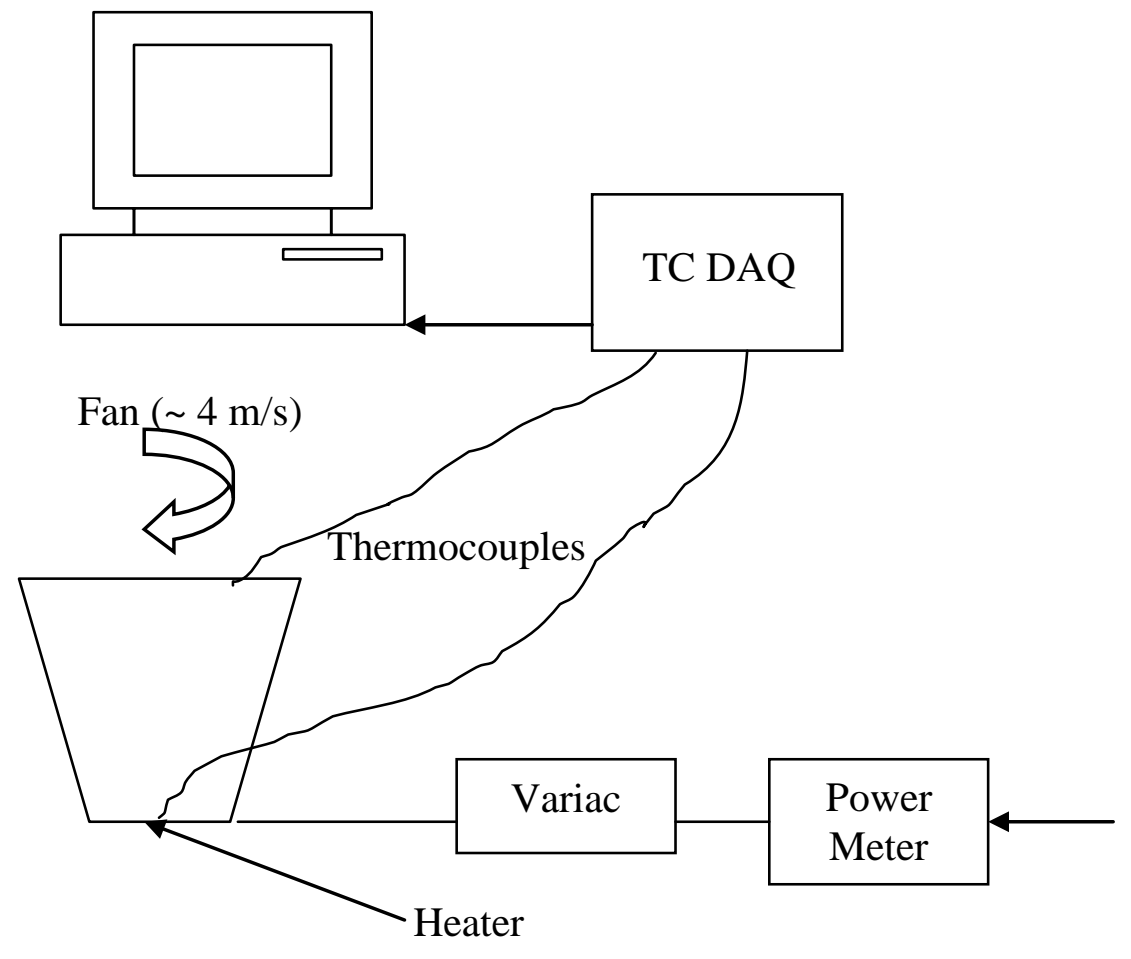




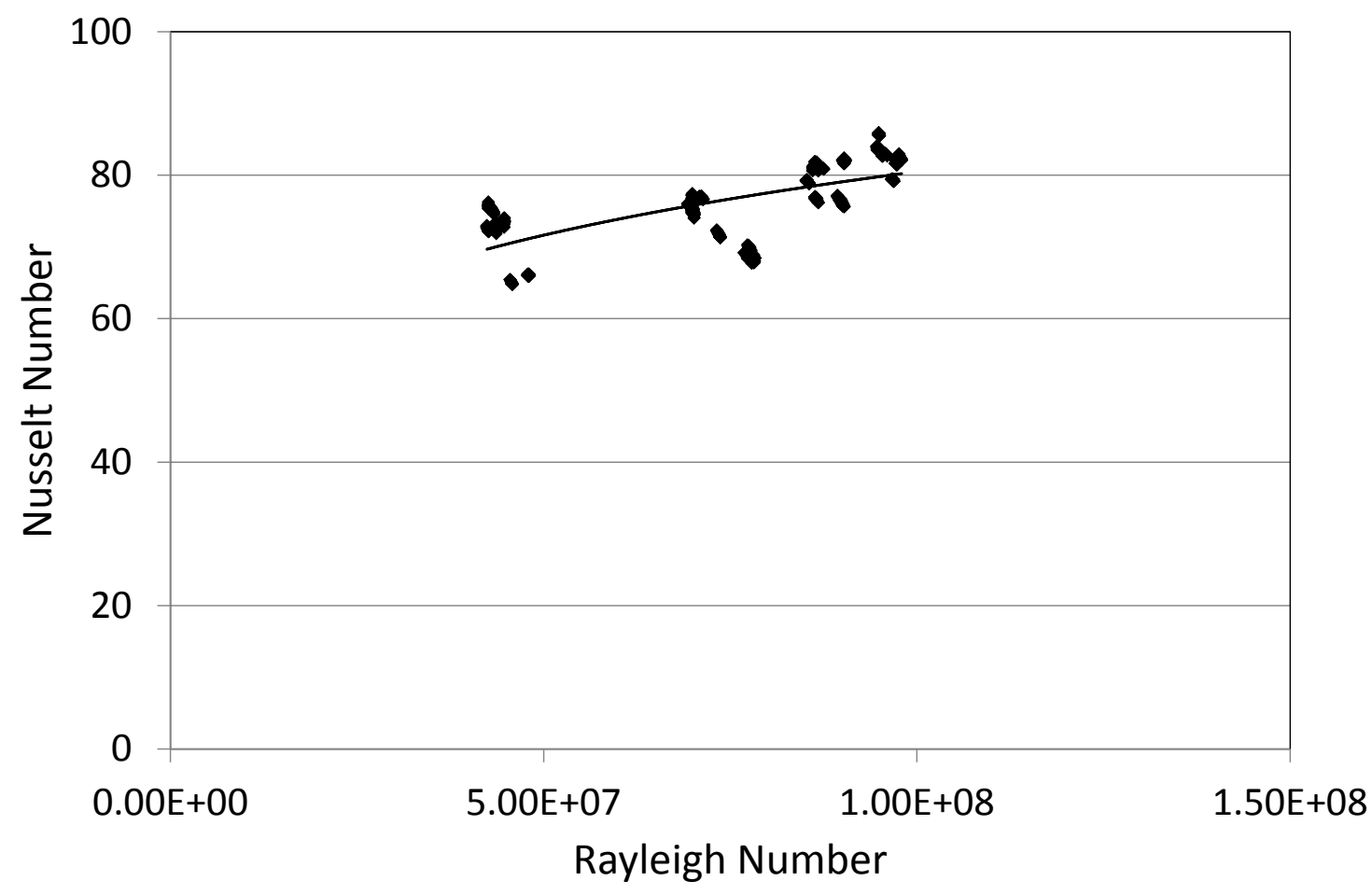




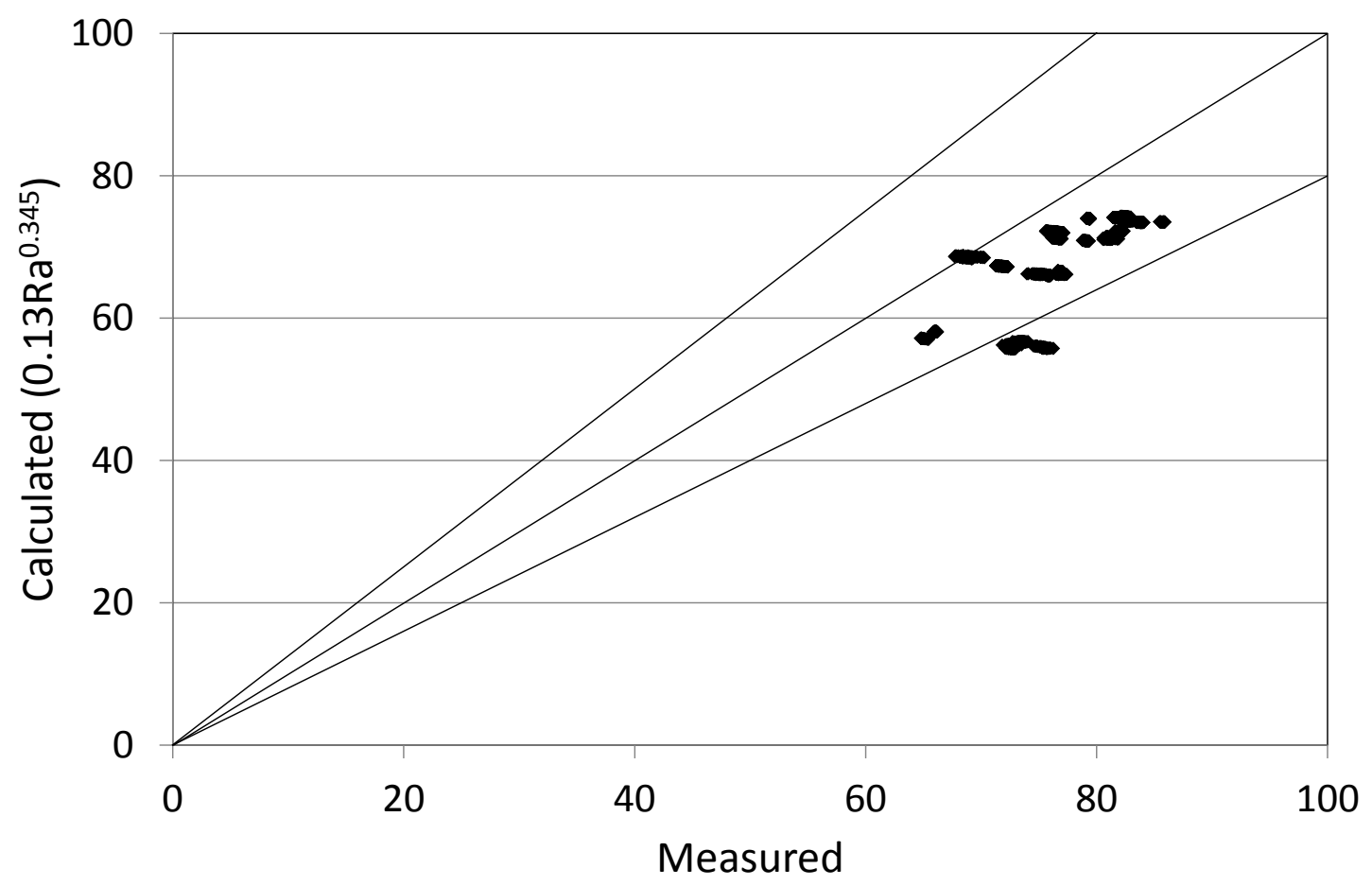

